\title{
Identités et compétences professionnelles dans les années 1920
}

Les professeurs de lycée, les « primaires » et l'inspection d'académie Professional Identities and Skills in the 1920s. Secondary School Teachers, Primary School Teachers and the School's Inspectorate Berufliche Identitäten und Kompetenzen in den Zwanziger Jahren. Die Gymnasiallehrer, die Grundschullehrer und das Oberschulamt Identidades y competencias profesionales en los años 1920. Los profesores de instituto, los de "primaria" y la inspección de academia

\section{Yves Verneuil}

\section{(2) OpenEdition Journals}

Édition électronique

URL : https://journals.openedition.org/histoire-education/1827

DOI : 10.4000/histoire-education. 1827

ISSN : 2102-5452

Éditeur

ENS Éditions

Édition imprimée

Date de publication : 1 janvier 2009

Pagination : 43-66

ISBN : 979-2-7342-1142-6

ISSN : 0221-6280

Référence électronique

Yves Verneuil, «Identités et compétences professionnelles dans les années 1920 », Histoire de l'éducation [En ligne], 121 | 2009, mis en ligne le 01 janvier 2014, consulté le 20 mai 2021. URL : http:// journals.openedition.org/histoire-education/1827 ; DOI : https://doi.org/10.4000/histoire-education. 1827 


\title{
Identités et compétences professionnelles dans les années 1920
}

\author{
Les professeurs de lycée, les "primaires" \\ et l'inspection d'académie
}

\section{Yves VERNEUIL}

Les débats autour d'un épisode apparemment mineur, l'élargissement, en 1925, de l'accès à l'inspection académique à des personnels issus de l'enseignement primaire, sont un bon révélateur des tensions corporatives qui compliquent la réalisation de l'école unique dans la France de l'entre-deux-guerres. L'épisode témoigne également de la crispation identitaire des professeurs de lycée face à des primaires dont ils jugent la culture inférieure ${ }^{1}$ et considèrent, par conséquent, les prétentions comme abusives. Dans la mesure où les professeurs de lycée vont, en outre, tirer argument de l'ouverture de l'inspection académique aux primaires pour exiger la suppression des inspections par les inspecteurs d'académie, cet épisode met également à jour, par-delà la défiance des professeurs de lycée envers "l'Administration ", leur profond attachement à la culture disciplinaire, vue comme un signe de compétence professionnelle. Ainsi se trouvent mis en évidence quelques éléments de l'identité professionnelle des professeurs de lycée, et spécialement de ceux qui prétendent exprimer la quintessence du corps, les professeurs agrégés.

Ces enjeux identitaires expliquent le caractère passionnel de la réaction des professeurs de lycée. Ceux-ci remettent d'autant plus vivement en cause

1 Cf. Viviane Isambert-Jamati, "Les primaires, ces incapables prétentieux ", Revue française de pédagogie, $\mathrm{n}^{\circ} 73,1985$, p. 57-65. 
le décret du 27 janvier 1925 élargissant l'accès des inspecteurs primaires aux fonctions d'inspecteur d'académie ${ }^{2}$, qu'il vient après la circulaire du 29 septembre 1924 qui, à leur grand dam ${ }^{3}$, a placé les professeurs des classes élémentaires sous le contrôle des inspecteurs primaires. Par conséquent, le décret du 27 janvier 1925 peut donner l'impression aux professeurs de lycée que les pouvoirs publics sont en train de réaliser la mise en place de l'école unique dans le sens voulu par les primaires, c'est-à-dire dans celui de la sujétion des secondaires, prélude à leur éviction.

À vrai dire, dès le congrès mixte qui, en 1904, avait réuni représentants des organisations primaires et délégués des amicales de professeurs de l'enseignement secondaire, de nombreux professeurs de lycée avaient été effrayés par les revendications des primaires : le thème de l'» égalité devant l'instruction " n'allait-il pas faire peser des menaces non seulement sur les classes élémentaires des lycées, mais également sur le premier cycle de l'enseignement secondaire, au profit des écoles primaires supérieures ? Cette inquiétude avait d'ailleurs largement contribué à la création de la Fédération nationale des professeurs de lycée et du personnel de l'enseignement secondaire féminin, en $1905^{4}$. L'identité des professeurs de l'enseignement secondaire s'affirmait donc contre les "primaires".

Après la guerre, le mouvement en faveur de l'« école unique " est relancé par les Compagnons de l'Université nouvelle. En 1922, sous couvert d'un pseudonyme, le directeur de l'enseignement primaire, Paul Lapie, publie dans la Revue pédagogique un article sur ce sujet ${ }^{5}$. La victoire électorale du Cartel des gauches, en 1924, parait sonner l'heure des réalisations. Constituée par Edouard Herriot, une Commission pour l'école unique est réunie par le ministre de l'Instruction publique François-Albert. La Fédération nationale des professeurs de lycée (qui se transforme, en 1925, en Syndicat national des professeurs de lycée et

2 Le décret du 27 janvier 1925 est souvent appelé décret Lapie, du fait du rôle joué par le directeur de l'enseignement primaire.

3 Cf. Bruno Garnier, Les Combattants de l'école unique, Lyon, INRP, 2008, p. 248-252. Bruno Garnier a utilisé le dossier Archives nationales (désormais AN) $\mathrm{F}^{17}$ 13951. Il faudrait compléter par le dossier $\mathrm{AN} \mathrm{F}^{17} 17500$, qui évoque directement les réactions des professeurs des classes élémentaires. Ceux-ci sont soutenus par la Fédération nationale des professeurs de lycée (à laquelle appartient la Société des professeurs des classes élémentaires).

$4 C f$. Yves Verneuil, "Les professeurs de l'enseignement secondaire au début du XX $\mathrm{X}^{\mathrm{e}}$ siècle : entre corporatisme et esprit de catégorie ", Carrefours de l'éducation, n 17, janvier-juin 2004, p. 102-117. Antoine Prost, L'Enseignement en France, 1800-1867, Paris, A. Colin, 1968, p. 407. 
du personnel de l'enseignement secondaire féminin 6 ) conteste la composition de cette commission. Au vrai, elle a peur que le mouvement ne débouche sur une " amputation " de l'enseignement secondaire. Certes, tous les professeurs de l'enseignement secondaire ne sont pas hostiles au mouvement pour l'école unique $^{7}$. Mais la défiance envers les "primaires " est avivée par la question de la revalorisation des traitements, que l'inflation d'après-guerre a rendue cruciale. Au sein du Comité d'entente universitaire qui s'est constitué pour tâcher de constituer une échelle commune de traitements, les professeurs de l'enseignement secondaire ont l'impression de se faire déborder par la masse des " primaires "; comme si, à leurs yeux, le nombre l'emportait sur la qualité. Les agrégés, en particulier, ne peuvent admettre, en 1925, que ce soit un inspecteur primaire, Riquet, qui négocie avec les pouvoirs publics pour l'ensemble du monde enseignant. Cela leur paraît être le monde à l'envers ${ }^{8}$.

Ce contexte éclaire la réaction des professeurs de lycée envers le décret Lapie. La discussion qui s'est ensuivie les a conduits à discuter du rôle des inspecteurs d'académie. Ainsi se trouve mis en évidence un autre aspect de leur identité. À sa naissance, leur mouvement amicaliste s'est en effet dressé non seulement contre les primaires, mais aussi contre le "despotisme administratif ". Les professeurs supportent mal le contrôle pédagogique du proviseur et de l'inspecteur d'académie. Cet aspect s'était déjà manifesté, en 1914, lors de la création de la Société des agrégés de l'enseignement secondaire : les membres agrégés de l'administration de l'Instruction publique avaient été admis, mais sans droit de vote. En 1923, cependant, une évolution s'est dessinée au sein de la Société des agrégés : celle-ci, qui prend alors le titre de Société des agrégés de l'Université, décide d'admettre à parité de droit les membres de l'administration (ainsi que ceux de l'enseignement supérieur) ${ }^{9}$. Cette évolution, qui parait marquer une alliance des agrégés et des inspecteurs d'académie, pose néanmoins la question des compétences professionnelles : les compétences administratives sont-elles

$6 \quad C f$. Jacques Girault, Instituteurs, professeurs, une culture syndicale dans la société française (fin XIX ${ }^{e}$ siècle-XX ${ }^{e}$ siècle), Paris, Publications de la Sorbonne, 1996.

7 Par exemple, Aimé Berthod, qui devient en 1919 vice-président de la Fédération nationale des professeurs de lycée, puis, en 1920, président de la Société des agrégés, avait publié dès 1906 un opuscule sur le sujet : Primaire et secondaire, pourquoi et comment il faut unifier l'enseignement, Poligny, édition des Annales de la Jeunesse laïque, 1906, 71 p.

8 Cf. Yves Verneuil, Les Agrégés. Histoire d'une exception française, Paris, Belin, coll. " Histoire de l'éducation ", 2005, p. 162.

9 Cf. Yves Verneuil, La Société des agrégés, de sa fondation à nos jours, thèse de doctorat, sous la dir. de Françoise Mayeur, Université Paris IV, 2001. 
les mêmes que les compétences professorales ? Ce sont ces équivoques que vont mettre à jour les débats concernant le décret Lapie. La double défiance des agrégés envers les primaires et envers le contrôle des inspecteurs d'académie va finalement leur interdire de dégager une solution autre que le refus pur et simple.

\section{I - Le recrutement des inspecteurs d'académie : réglementation et appréciations}

\section{1 - L'évolution du recrutement des inspecteurs d'académie}

Le décret du 17 mars 1808 organisant l'Université impériale n'impose pas de condition de titre à la nomination des inspecteurs d'académie. C'est l'ordonnance du 27 septembre 1832 qui prescrit le recrutement d'agrégés. En 1850, la loi Falloux supprime cependant cette exigence, réduite à la licence ou à dix ans d'exercice, et ouvre l'accès de la fonction aux inspecteurs primaires. Ces dispositions sont supprimées par la Troisième République : la loi du 30 octobre 1886 abroge en effet le titre premier de la loi du 15 mars 1850. De ce fait, aucun texte ne fixe plus de condition de recrutement pour les inspecteurs d'académie. Selon Paul Bert, toutefois, "l'administration a pris pour règle, il y a quelques années, de ne nommer inspecteurs d'académie que des agrégés ou des docteurs ès lettres ou ès sciences ${ }^{10}$. Le Nouveau dictionnaire de pédagogie et d'instruction primaire précise encore, en 1911 : "En fait, les inspecteurs d'académie sont choisis parmi les agrégés et les professeurs chargés de cours des lycées " ${ }^{11}$. Les ministres s'abstiennent donc de prendre les inspecteurs d'académie parmi les membres de l'enseignement primaire, alors même que c'est aux affaires de cet enseignement que ceux-ci consacrent la plus grande part de leur activité. La raison en est que l'inspecteur d'académie exerce aussi sa surveillance sur les lycées et les collèges, si bien que les autorités estiment que, pour faire bonne figure, il doit être muni de grades élevés, qui fassent de lui au moins l'égal des professeurs de lycée.

Le monopole des professeurs de lycée est cependant brisé par le décret du 14 février 1916, qui ouvre l'inspection académique aux inspecteurs primaires

10 Paul Bert, "Directeur départemental de l'enseignement primaire ", in Ferdinand Buisson (dir.), Dictionnaire de pédagogie et d'instruction primaire, Paris, Hachette, 1887, $1^{\mathrm{re}}$ partie, t. 1, p. 172.

11 "Inspecteurs d'académie ", in Ferdinand Buisson (dir.), Dictionnaire de pédagogie et d'instruction primaire, Paris, Hachette, 1911, consulté en ligne à l'adresse :

<http://www.inrp.fr/edition-electronique/lodel/dictionnaire-ferdinand-buisson/>. 
et aux directeurs d'école normale : outre les agrégés et les docteurs, pourront être recrutés des titulaires du certificat d'aptitude à l'inspection primaire et à la direction d'écoles normales, du moins s'ils sont pourvus de la licence. Le rapport ministériel du 7 février 1916 justifie cette exigence : "Comme l'inspecteur d'académie est, dans son département, le chef de l'enseignement secondaire, on exige des membres de l'enseignement primaire qui sollicitent cet emploi le titre qui leur permettrait d'enseigner dans un lycée ou un collège " ${ }^{12}$. L'article 4 du décret précise néanmoins que " les fonctionnaires qui auront suppléé les inspecteurs d'académie mobilisés pendant la guerre pourront être délégués dans les fonctions d'inspecteur d'académie même s'ils ne possèdent pas les titres requis par l'article 3 ".

Ce décret suscite l'indignation de la Fédération nationale des professeurs de lycée, scandalisée que des inspecteurs primaires dépourvus de la licence puissent profiter de la mobilisation d'inspecteurs d'académie agrégés. En pratique, toutefois, ce décret est peu appliqué, puisque deux inspecteurs primaires seulement en bénéficient. Et la protestation des professeurs de lycée n'est sans doute pas étrangère à la parution du décret du 14 mars 1919, qui limite à une sur dix les nominations d'inspecteurs d'académie provenant de l'enseignement primaire ${ }^{13}$.

En 1925, un nouveau pas est franchi. Le décret du 27 janvier étend le recrutement des inspecteurs d'académie aux titulaires du certificat d'aptitude à l'inspection primaire et à la direction d'école normale qui sont en même temps titulaires du certificat d'aptitude au professorat des écoles normales et des écoles primaires supérieures ${ }^{14}$. La licence n'est donc plus une condition sine qua non. Aucun quota n'est plus fixé.

\section{2 - Les réactions au décret Lapie}

Du côté des primaires, c'est évidemment la satisfaction qui domine, comme l'exprime L'Information scolaire : " Depuis longtemps, directeurs d'écoles normales et inspecteurs primaires réclamaient une modification de ce régime [les décrets limitatifs de 1916 et de 1919]. Ils viennent d'obtenir satisfaction. [...] Tout le personnel de l'enseignement primaire se réjouira de cette réparation

12 Bulletin administratif du ministère de l'Instruction publique (dorénavant BAMIP), Paris, Imprimerie nationale, 1916, p. 231.

13 BAMIP, 1919, p. 450.

14 Ce décret n'est pas mentionné par Jacques Effroy, L'Inspection académique en résidence départementale. Hier, aujourd'hui, demain, thèse, sous la dir. de Josette Poinssac, Université Paris VIII, 1995. 
depuis longtemps attendue ${ }^{15}$. Ce terme de " réparation " a l'art d'exaspérer les secondaires, également inquiets des conclusions assimilatrices que les directeurs et directrices d'écoles normales tirent du décret :

"Osons dire avec quelque fierté que c'est un succès moral et un accroissement de la dignité de notre fonction que l'emploi d'inspecteur d'académie nous soit désormais accessible par assimilation du professorat avec la licence ${ }^{16}$.

Aux yeux des directeurs et directrices d'écoles normales, dans la mesure où les fonctions d'inspecteur d'académie, ayant cessé d'être réservées aux agrégés et aux docteurs, avaient été ouvertes aux licenciés, il était juste que les titulaires du professorat des écoles normales puissent y avoir accès, puisque cet examen n'était pas moins que la licence " une preuve suffisante de culture, surtout quand il se double du certificat d'aptitude à l'inspection primaire qui sanctionne l'aptitude pédagogique $"{ }^{17}$.

Dans ces conditions, on pourrait être surpris de la réaction de l'Association des inspecteurs primaires munis du professorat, qui réunit les inspecteurs primaires concernés par le décret Lapie, à l'exclusion des inspecteurs primaires sortis du rang. Issu d'une scission de l'Association des inspecteurs primaires intervenue en $1924^{18}$, ce groupement fait en effet savoir que le décret ne correspond pas à ses attentes : il aurait plutôt souhaité la limitation des compétences de l'inspecteur d'académie et la création de la fonction de directeur départemental de l'enseignement primaire, fonction qui aurait été réservée aux fonctionnaires issus de cet enseignement. Dans une lettre à la Société des agrégés, Dussuel, président de l'Association des inspecteurs primaires anciens professeurs, précise que seulement un membre sur sept de son organisation est d'accord avec le décret du 27 janvier $1925^{19}$.

Du côté des professeurs de lycée, la réaction hostile est unanime. La Fédération nationale des professeurs de lycée avait déjà émis de vives protestations contre le décret du 14 février 1916. Par patriotisme, rappelait-elle, elle avait mis en veille ses revendications pendant la durée de la guerre, mais « cette

15 L'Information scolaire, $\mathrm{n}^{\circ} 5$, février 1925, p. 92.

16 Bulletin de l'Association amicale des directeurs et directrices d'écoles normales, $\mathrm{n}^{\circ} 18$, février-juin 1925, p. 12.

17 Ibid., $\mathrm{n}^{\circ} 17$, juillet 1924-janvier 1925, p. 8.

18 Les inspecteurs primaires munis du professorat veulent une indemnité spécifique, tandis que les inspecteurs primaires sortis du rang (anciens instituteurs) tiennent au contraire pour un traitement unique lié à la fonction.

19 L'Agrégation, n 6, avril 1926, p. 33-37. 
discrétion patriotique ne saurait aller jusqu'au sacrifice des intérêts de l'enseignement. [...] Le décret est nuisible au bon fonctionnement de l'administration, préjudiciable à l'enseignement secondaire tout entier ${ }^{20}$. Selon Albert Fédel, son président, ce décret du 14 février 1916 reproduisait, pour les conditions du recrutement des inspecteurs d'académie, les dispositions de la loi Falloux, dont la marque était le mépris des grades et des titres pour mieux asseoir le despotisme administratif. L'ouverture de l'inspection d'académie aux primaires était donc une mesure antirépublicaine. Au demeurant, le Conseil supérieur de l'Instruction publique n'avait pas été consulté (cependant, le pourvoi auprès du Conseil d'État formé sur ce motif n'aboutit pas).

En 1925, la Fédération n'a pas changé d'avis ${ }^{21}$. Son opposition au décret Lapie est soutenue par la Société des professeurs de français et de langues anciennes (dite Franco-ancienne), qui y voit un nouvel épisode de la "guerre à l'enseignement secondaire "22. À la Société des agrégés, le décret Lapie soulève un véritable tollé. L'inspecteur d'académie Étienne L. Ferrère, membre du comité, est chargé d'exprimer l'indignation de la Société :

"Le décret du 27 janvier 1925 [...] est humiliant pour tous les professeurs agrégés de l'enseignement secondaire ; il est humiliant, même, pour les inspecteurs d'académie, qui sont encore agrégés en très grande majorité, et auxquels on va imposer le voisinage de collègues de deuxième zone $"^{23}$.

Si l'on s'en tient à son bulletin, l'Association amicale des inspecteurs d'académie ne paraît pourtant pas s'émouvoir du décret Lapie. En 1928 encore, son assemblée générale refuse de traiter de la question de leur recrutement : "Le président fait observer que les inspecteurs d'académie ayant diverses origines, il ne semble pas qu'une amicale, qui les réunit tous, soit qualifiée pour discuter de cette question " ${ }^{24}$. Divers indices montrent toutefois l'hostilité des inspecteurs d'académie agrégés à l'accueil des primaires. Devant la commission tripartite formée par l'administration qui évoquait notamment la question du recrutement des inspecteurs d'académie, Maurellet, président de l'Association amicale des inspecteurs d'académie, avait demandé que les professeurs et pro-

20 Bulletin officiel de la Fédération nationale des professeurs de lycée et du personnel de l'enseignement secondaire féminin (désormais BOFNPL), n 98, mars-avril-mai 1916, p. 185.

21 BOFNPL, $\mathrm{n}^{\circ} 168,1925$, p. 456.

22 Bulletin de la Société des professeurs de français et de langues anciennes de l'enseignement secondaire, $\mathrm{n}^{\circ} 35$, mars 1925 , p. 4.

23 L'Agrégation, $\mathrm{n}^{\circ}$ 6, mai-juin 1925, p. 35.

24 Bulletin de l'Association amicale des inspecteurs d'académie, $\mathrm{n}^{\circ}$ 9, janvier 1928, p. 3. 
viseurs de lycée soient consultés ${ }^{25}$. Selon les confidences de Ferrère, il avait par ailleurs organisé une consultation officieuse des adhérents de son association ; une grande majorité d'entre eux avaient fait savoir qu'ils n'étaient pas partisans d'une plus grande accession des primaires à l'inspection d'académie. Surtout, la question de la revalorisation des traitements va finalement conduire l'amicale à se prononcer nettement. Elle a obtenu la parité des traitements de fin de carrière des inspecteurs d'académie avec ceux des chefs de bureau et des chefs de service départementaux. Mais, comme elle tient au maintien de l'indemnité d'agrégation, le traitement des inspecteurs d'académie non agrégés se trouve inférieur d'autant. Ces derniers se montrent mécontents, mais le président de l'association répond, en 1927, qu'» il est équitable que les grades conquis par les inspecteurs d'académie leur assurent une indemnité supplémentaire ; [que] le recrutement des inspecteurs d'académie dépend en grande partie de cette indemnité ${ }^{26}$ : ce qui revient à déclarer qu'il souhaite le recrutement d'agrégés. En 1930, le secrétaire général du Syndicat des directeurs et directrices d'école normale, qui constate que cette situation financière est un obstacle à l'élévation du traitement des fonctionnaires subordonnés aux inspecteurs d'académie, propose l'institution d'un traitement unique des inspecteurs d'académie sous réserve qu'il ne soit plus nommé que des agrégés. L'amicale des inspecteurs d'académie, qui ne croit pas que tous les directeurs d'école normale verraient d'un bon œil la suppression du décret qui leur ouvre l'inspection académique, préfère redire son attachement à l'indemnité d'agrégation. La question est toutefois suffisamment sensible pour que soit convoquée une assemblée générale extraordinaire. Celle-ci décide la tenue d'un référendum, à la suite duquel l'amicale demande que tous les inspecteurs d'académie aient le traitement des chefs de bureau, l'indemnité d'agrégation devant cette fois être en sus : vœu dont la réalisation est douteuse. De toute façon, une seconde résolution est adoptée, qui demande que " désormais le recrutement des inspecteurs d'académie se fasse uniquement parmi les agrégés " ${ }^{27}$.

À cette date, la Société des agrégés comprend quarante-huit inspecteurs d'académie. Que ces membres de l'administration aient accepté de côtoyer leurs subordonnés dans une association corporative montre assez que la majorité

25 L'Information scolaire, $\mathrm{n}^{\circ} 4$, janvier 1925, p. 70.

26 Bulletin de l'Association amicale des inspecteurs d'académie, n 7, octobre 1927, p. 5.

27 Ibid., n 15, avril 1931, p. 2. 
des inspecteurs d'académie agrégés partagent les conceptions des professeurs agrégés à l'égard des " primaires ".

\section{II - Ces primaires « remuants et prétentieux » : la crispation identitaire des professeurs de l'enseignement secondaire}

Pour les agrégés, le décret Lapie est intolérable pour deux raisons : les primaires n'ont aucune compétence pour juger des qualités des professeurs de lycée ; il ne saurait être question, de toute façon, qu'un primaire soit placé au-dessus d'un agrégé.

La première raison est clairement exprimée par la section montpelliéraine de la Société des agrégés : "Les inspecteurs primaires ne sont qualifiés ni par leur préparation, ni par leur expérience professionnelle, pour venir inspecter les professeurs de l'enseignement secondaire $"{ }^{28}$. Les primaires ne possèdent pas la culture propre à l'enseignement secondaire - celle-ci s'exprimant avant tout par l'art de bien parler et de bien écrire. C'est pourquoi, quand L'Information scolaire se réjouit, au nom des primaires, qu'ait été supprimée l'exigence de la licence, "lien notoire avec l'enseignement secondaire " ${ }^{29}$, les professeurs de lycée se gaussent de cette impropriété. Aux yeux des agrégés, la licence paraissait déjà un bien mince vernis de culture secondaire. A fortiori les diplômes de l'enseignement primaire sont-ils inappropriés s'il s'agit d'inspecter des professeurs de l'enseignement secondaire. Les agrégés ne voient pas comment un inspecteur primaire pourrait juger et apprécier un cours dont il ne comprendrait pas la teneur. "Ainsi un inspecteur primaire [...] n'ayant jamais enseigné, ni même étudié le latin, le grec, l'anglais ou l'allemand, pourra être nommé inspecteur d'académie et, comme tel, juger et apprécier, par des notes qui influent sur leurs promotions et leur avancement, nos collègues agrégés de lettres, de grammaire, de langues vivantes, etc. ${ }^{30}$. En vérité, un agrégé ne doit être inspecté que par un agrégé, car, insiste la section d'Avignon, "la possession de l'agrégation [montre] toujours que l'on est familiarisé avec l'esprit et les méthodes qui règnent et doivent continuer à régner dans l'enseignement secondaire ${ }^{31}$. En effet, la différence entre les deux enseignements n'est pas

\footnotetext{
28 L'Agrégation, ${ }^{\circ}$ 6, mai-juin 1925, p. 50.

29 L'Information scolaire, $\mathrm{n}^{\circ}$ 5, février 1925, p. 92.

30 L'Agrégation, $\mathrm{n}^{\circ}$ 4, février-mars 1925, p. 12.

31 L'Agrégation, ${ }^{\circ}$ 5, avril 1925, p. 9.
} 
seulement une différence de contenu, elle est aussi une différence d'esprit. Le décret Lapie va donc instituer comme inspecteurs d'académie des inspecteurs primaires " radicalement incapables de juger, et même de comprendre les professeurs de l'enseignement secondaire qui vont se trouver placés sous leur autorité " 32 . Dans ces conditions, analysait déjà Albert Fédel en 1916, il y a lieu de craindre que l'inspecteur primaire indûment promu se comporte en despote non éclairé :

" $\mathrm{Si}$, comme il y a lieu de le penser, il tient à réagir contre l'impression d'incapacité qu'il donnera et à établir son autorité, il sera fatalement amené à dissimuler son insuffisance à l'aide de quelque discussion sur une question de méthode, ou à essayer d'imposer aux professeurs la théorie ou les habitudes pédagogiques qui lui sont chères $"^{33}$.

L'assimilation entre pédagogie et esprit primaire est caractéristique des professeurs de lycée ${ }^{34}$. Elle se double d'une assimilation entre pédagogie et despotisme qui remonte, chez les agrégés, au ministère Fortoul ${ }^{35}$. De ce fait, les inspecteurs primaires sont d'autant plus suspects de se comporter en petits chefs étrangers que leur compétence paraît bien limitée. Une anecdote rapportée par le bulletin de la Société des agrégés illustre cet état d'esprit. Elle porte sur le comportement d'un inspecteur primaire désigné pour présider le concours des bourses, qui sert aussi à entrer en sixième ou en cinquième ${ }^{36}$.

" [L'inspecteur primaire] n’a pas du tout été embarrassé de sa nouvelle mission, au contraire. N'avait-il pas dit, à la nouvelle du décret Albert (sic), organisant l'inspection des classes élémentaires : " Il n'est pas trop tôt qu'on aille voir ce qui se passe dans les lycées !... " ? Ce président a d'abord protesté contre la présence du proviseur. Il a fallu que ce dernier exhibât une lettre de l'inspecteur d'académie. Puis il a donné des "instructions " sur la correction du français et l'interrogation dans cette même matière à nos collègues agrégés, qui ont regimbé quelque peu ; il a prétendu les employer à interroger aussi en histoire ; ils ont décliné leur compétence et s'y sont refusés : scrupule qu'il n'a pas compris, ni (non plus que la plupart des siens) ne saurait comprendre ${ }^{37}$.

32 L'Agrégation, $\mathrm{n}^{\circ}$ 6, mai-juin 1925, p. 35.

33 BOFNPL, n 98, mars-avril-mai 1916, p. 188.

$34 C f$. Évelyne Hery, Les Pratiques pédagogiques dans l'enseignement secondaire au $20^{e}$ siècle, Paris, L'Harmattan, 2007, p. 63.

35 Cf. Yves Verneuil, Les Agrégés..., op. cit., p. 43-44.

36 Le décret du 9 janvier 1925 a institué un " régime unique pour l'attribution des bourses nationales dans les établissements publics du second degré ".

37 L'Agrégation, $n^{\circ}$ 7, juillet 1925, p. 7-8. 
L'anecdote témoigne de l'exaspération des agrégés à se trouver sous la coupe de personnes qu'ils situent à un niveau inférieur au leur dans la hiérarchie universitaire. À la suite de cet épisode, le comité de la Société des agrégés vote d'ailleurs, à propos de l'examen des bourses, un vœu par lequel il "proteste contre la subordination des professeurs et des proviseurs de lycée aux inspecteurs primaires ${ }^{38}$. Au vrai, cette question de la subordination est la raison la plus fondamentale du refus du décret Lapie. En 1916, Albert Fédel écrivait déjà : " Il serait vraiment trop commode de devenir le chef d'un professeur quand on ne pourrait pas être son collègue $" 39$. En 1925, le caractère insupportable de l'assujettissement à des primaires est crûment exprimé. Ainsi, pour le président de la Société des agrégés, Édouard Rey, " il est absolument impossible que des professeurs agrégés soient subordonnés à des inspecteurs primaires $"^{40}$. Les professeurs de l'enseignement secondaire s'en tiennent au principe qui semblait avoir été énoncé par le décret du 31 mai 1902 (qui avait exigé le titre d'agrégé pour la nomination au provisorat) : le chef ne saurait être inférieur en grade et en titre à ses subordonnés. Lors de l'audience que lui a accordée le directeur de l'enseignement secondaire, le 12 mars 1925, la Société des agrégés expose nettement qu'" un agrégé souffrira difficilement d'être noté par un chef pourvu de grades et de titres entièrement différents ". Mais Paul Lapie "répond que cela lui paraît être un préjugé "41.

À la critique du préjugé en faveur des titres, la Société des agrégés répond par la dénonciation de la subversion des valeurs : car non seulement le concours de l'agrégation répond à l'esprit de l'enseignement secondaire, mais encore les titres des professeurs de l'enseignement secondaire sont supérieurs à ceux des maîtres de l'enseignement primaire.

"Le Comité de la Société des agrégés, estimant que les diplômes universitaires marquent une hiérarchie des valeurs, et non pas seulement des spécialisations pédagogiques. [...] Estimant d'autre part que l'agrégation est, dans les limites de l'enseignement secondaire, le titre qui [...] confère le premier rang dans la hiérarchie des professeurs, [...] dénie à tout inspecteur, non pourvu de l'agrégation, le droit d'inspecter et de noter les professeurs agrégés ${ }^{42}$.

38 Ibid., p. 8.

39 BOFNPL, n 98, mars-avril-mai 1916, p. 187.

40 L'Agrégation, $\mathrm{n}^{\circ}$ 4, février-mars 1925, p. 7.

41 L'Agrégation, $\mathrm{n}^{\circ}$ 5, avril 1925, p. 4.

42 L'Agrégation, $\mathrm{n}^{\circ} 1$, octobre 1925, p. 7. 
À qui objecterait que cet attachement à la hiérarchie témoigne d'un esprit peu démocratique, la Fédération nationale des professeurs de lycée a déjà répondu, en 1916, dans une formule qui a souvent été reprise par la suite, que "le nivellement par le bas n'a rien de commun avec la démocratie "43.

L'appel à la mobilisation générale " pour que nous ne soyons pas, malgré nous, "primarisés " " ${ }^{44}$, correspond à l'idée que les professeurs de l'enseignement secondaire subissent une véritable "invasion des primaires " ${ }^{45}$. Lors de l'assemblée générale de la Société des agrégés de 1927, Goulard, sous les applaudissements, "affirme la nécessité de défendre l'enseignement secondaire et de barrer la route aux primaires " ${ }^{46}$. Ce langage révèle une mentalité de citadelle assiégée : ainsi É. L. Ferrère écrit-il qu'avant même le décret Lapie, les primaires étaient déjà " dans la place et y fortifiaient de plus en plus leurs positions ${ }^{47}$.

La défense de la place masque en fait la lutte pour " les places". La seule justification qui a été donnée au décret par Paul Lapie devant la Société des agrégés n'est-elle pas qu'" on ne peut condamner les primaires d'élite à avoir des situations toujours inférieures " ${ }^{48}$ ? Selon Théodore Suran, qui préside la Franco-ancienne : "Le décret abaissant (sic) encore l'inspection académique ne se colore [...] d'aucune ombre de prétexte : les appétits aiguisés ne veulent plus être rationnés, il leur faut tout. Demain, on leur livrera l'Inspection générale. C'est la guerre à l'enseignement secondaire, la guerre de gens qui ne veulent pas travailler pour l'avenir de leur pays, mais pour la satisfaction immédiate de leur arrivisme personnel $"{ }^{49}$. Dans le même numéro de la Revue de la Francoancienne est racontée l'anecdote suivante :

" Je causai dernièrement avec un inspecteur primaire qui est un des plus acharnés contre nous ; il n'a pas été long à m'en donner la raison de dernière la tête : "C'est toujours aux secondaires qu'on donne les meilleures places ". [...] Ce mot de places est tout un poème. Il n'est pas question de fonctions ou de capacités, mais de places, comme il était question sous l'Ancien Régime de bénéfices ou de prébendes. C'est de la jalousie pure. [...]

43 BOFNPL, n 98, mars-avril-mai 1916, p. 187.

44 L'Agrégation, n 4 , février-mars 1925, p. 11-12.

45 L'Agrégation, $\mathrm{n}^{\circ}$ 8, juillet 1927, p. 40.

46 L'Agrégation, n 6, avril-mai 1927, p. 17.

47 L'Agrégation, $\mathrm{n}^{\circ}$ 6, mai-juin 1925, p. 34.

48 L’Agrégation, n 4, février-mars 1925, p. 4.

49 Bulletin de la Société des professeurs de français et de langues anciennes de l'enseignement secondaire, $\mathrm{n}^{\circ} 35$, mars 1925, p. 4. 
"Qui nous délivrera de la malfaisante influence de ces jaloux ? Si les futurs instituteurs et professeurs d'écoles primaires supérieures et d'écoles normales étaient obligés de passer par le lycée ou le collège (la voilà bien, l'école unique, ce n'est pas nous qui la redoutons !), ils accéderaient à leur guise où ils voudraient. Ils préfèrent la substitution à la préparation ; ce qu'ils veulent, ce sont des places ${ }^{50}$.

C'est donc du côté de l'enseignement secondaire que se situent les valeurs républicaines, puisque les primaires sont suspects de préférer les sourdes manigances au véritable travail. Ferrère résume certainement l'opinion dominante des professeurs de l'enseignement secondaire quand il écrit : "Le décret du 27 janvier 1925 [...] a été arraché au ministre par des primaires remuants et prétentieux $"$.

Il distingue cependant de ces arrivistes la majorité des inspecteurs primaires, qui, selon lui, ont reçu avec le décret Lapie un cadeau qu'eux-mêmes ne désiraient pas. De fait, Dussuel, le président de l'Association des inspecteurs primaires anciens professeurs écrit à la Société des agrégés qu'il est d'accord avec l'idée selon laquelle celui qui veut inspecter des agrégés doit d'abord passer l'agrégation ; qu'il approuve lui aussi le principe selon lequel l'inspecteur doit avoir les titres de l'inspecté ${ }^{52}$. Ce principe, cependant, peut aussi servir à dénier aux inspecteurs d'académie agrégés le droit d'inspecter les primaires, dont les diplômes sont autres. Son application stricte entraînerait donc une réorganisation administrative qui limiterait le rôle de l'inspection académique à l'enseignement secondaire. Cette réforme, il est vrai, aurait aussi pour effet de résoudre la question de l'accès des primaires à l'inspection d'académie. C'est pourquoi elle a été envisagée par les professeurs de lycée.

\section{III - Les agrégés face aux inspecteurs d'académie}

\section{1 - Profiter des circonstances pour limiter le rôle de l'inspecteur d'académie ?}

L'idée de dissocier contrôle sur l'enseignement primaire et contrôle sur l'enseignement secondaire n'est pas nouvelle. En 1882, Paul Bert avait proposé de créer la fonction de directeur départemental de l'enseignement primaire,

50 Ibid., p. 30-31.

51 L'Agrégation, n 6, mai-juin 1925, p. 35.

52 L'Agrégation, $\mathrm{n}^{\circ}$ 6, avril 1926, p. 33. Cette opinion reflète aussi l'hostilité des professeurs d'école primaire supérieure (munis d'un certificat d'aptitude spécifique) à être inspectés par des inspecteurs primaires sortis du rang. 
fonction qui aurait été attribuée à des fonctionnaires issus de l'enseignement primaire, parallèlement à l'institution d'inspecteurs de l'enseignement secondaire, un pour les lettres, l'autre pour les sciences, qui auraient été établis au chef-lieu de chaque académie ${ }^{53}$. Lors d'une réunion de la Ligue de l'enseignement, à Rennes, en 1898, ce projet avait de nouveau été évoqué par le directeur de l'enseignement primaire, Charles Bayet, avec l'appui du ministre Léon Bourgeois. En 1924, le ministre de Jouvenel avait proposé un projet semblable, qui aurait abouti à la départementalisation de l'inspection primaire et à la régionalisation de l'inspection académique ${ }^{54}$. L'opposition du directeur de l'enseignement primaire, Paul Lapie, et de son collègue de l'enseignement secondaire, Raymond Thamin, qui avaient objecté la nécessité de maintenir un fonctionnaire départemental assurant la coordination entre les deux enseignements, avait conduit au retrait du projet.

L'institution de la fonction de directeur départemental de l'enseignement primaire correspond néanmoins à une réclamation des inspecteurs primaires, dont l'association a voté un vœu en ce sens en 1923. Ces derniers supportent de plus en plus difficilement l'autorité d'inspecteurs d'académie venus de l'enseignement secondaire, à la culture fort différente - et parfois à la condescendance marquée. De fait, recteurs et inspecteurs d'académie, passés par l'enseignement secondaire, donnent souvent des appréciations sans aménité de leurs subordonnés et de leur culture " primaire ", comme le suggère ce jugement porté en 1909 par le recteur sur l'inspecteur primaire de la Châtre : " J'ai lu quelques-uns des articles de M. D. ; ils dénotent un effort de pensée qui n'est pas négligeable et qu'il ne faut pas décourager... même chez un inspecteur primaire ${ }^{55}$. Même état d'esprit de la part de Paul Bréhat, inspecteur honoraire : dans son récit sur le métier d'inspecteur d'académie, publié en 1934, il ironise sur les propriétaires qui feignent d'ignorer le nom de leurs métayers, mais lui même ne s'adresse pas aux instituteurs autrement que par un condescendant " mon ami "; et son analyse de l'examen du brevet reprend la critique traditionnelle de l'encyclopédisme des primaires ${ }^{56}$.

53 Jacques Effroy, op. cit., p. 84-85.

54 Association amicale des inspecteurs d'académie. Bulletin annuel, 1924, p. 8.

55 Cité par Jean Ferrier, Les Inspecteurs des écoles primaires, 1835-1995, Paris, L'Harmattan, p. 113.

56 Paul Bréhat, Un beau métier. Grandeur et servitude universitaires, Verdun, Impr. et librairie Marchal, 1934. 
C'est pour des raisons parallèles, mais inverses, à celles des inspecteurs primaires, que la Fédération nationale des professeurs de lycée s'est ralliée à l'idée de séparer contrôle sur les établissements secondaires et direction départementale de l'enseignement primaire : son ralliement est en effet consécutif à l'ouverture de l'inspection d'académie aux primaires. Dès le décret de 1916, son président, Albert Fédel, pose clairement l'alternative : soit l'inspection des professeurs de lycée leur est conservée, et il faut alors des inspecteurs d'académie agrégés ou docteurs ; soit on recrute des primaires, et les inspecteurs d'académie ne doivent inspecter que les établissements primaires, le recteur seul notant et inspectant les professeurs de lycée et de collège. En 1919, la Société des agrégés émet un vœu allant dans le même sens et demande l'institution d'inspecteurs régionaux de l'enseignement secondaire ${ }^{57}$. En 1925, suite au décret Lapie, la Fédération nationale souhaite que désormais l'inspecteur d'académie soit " exclusivement le directeur départemental de l'enseignement primaire " 58 . Quant à la Société des agrégés, elle demande que les attributions touchant à l'enseignement secondaire soient enlevées aux inspecteurs d'académie départementaux et qu'il soit établi auprès du recteur deux inspecteurs d'académie spécialisés, qui auraient la surveillance de l'enseignement secondaire ${ }^{59}$.

La concordance des vœux des inspecteurs primaires et des professeurs de lycée témoigne du désir de chaque ordre d'enseignement d'être maître chez lui. Alors que le contexte est à la réflexion sur l'école unique, les plus éminents représentants de chacun des deux ordres d'enseignement ne pensent qu'à les développer de façon autonome.

La communauté de vue entre inspecteurs primaires et professeurs de lycée aurait pu favoriser la réalisation du projet. Un agrégé conservateur comme Pierre Couissin fait d'ailleurs remarquer que, pour une fois que les agrégés ont affaire à des primaires qui ont des vues raisonnables (des primaires qui déclinent l'honneur d'inspecter des agrégés), ils doivent saisir l'occasion. Cependant le projet rencontre toujours l'hostilité du directeur de l'enseignement primaire, Paul Lapie. À la délégation de la Société des agrégés, il ne cache pas son opposition, " car ce qu'il veut assurer, c'est la liaison entre les deux enseignements ; or la mesure proposée ne ferait que perpétuer le divorce "60.

57 L'Agrégation, n 5, mars 1919, p. 5.

58 Quinzaine universitaire, 15 février 1925, p. 1.

59 L'Agrégation, $\mathrm{n}^{\circ}$ 5, avril 1925, p. 4.

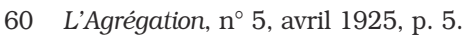


Aux yeux de la Société des agrégés, le recteur pourrait assurer cette liaison, mais, pour Paul Lapie, celle-ci ne serait pas suffisante. Une autre opposition provient de l'association des inspecteurs d'académie, qui refuse une diminution des attributions de ses membres ${ }^{61}$.

De toute façon, à la Société des agrégés, le projet d'instituer des inspecteurs régionaux, qui était passé inaperçu en 1919, ne fait plus l'unanimité en 1925. Des sections protestent et l'assemblée générale de 1926 ne parvient pas à se décider. Finalement, l'année suivante, elle repousse l'idée d'organiser une inspection régionale secondaire. Trois arguments principaux ont conduit à cette décision.

Certains agrégés, tel Maurice Weber, compagnon de l'Université nouvelle, sont favorables à un rapprochement des enseignements primaire et secondaire. Or le projet " empêcherait la coordination du primaire et du secondaire, au moment où il est question de la rendre plus étroite ", dénonce la section de Valence ${ }^{62}$. Si l'on veut que l'école unique ne se réalise pas aux dépens des lycées et de leur personnel, il faut maintenir l'inspecteur d'académie dans son rôle - mais exiger qu'il reste un agrégé. L'exemple du concours des bourses ne montre-t-il pas qu'il importe que la coordination entre enseignement primaire et enseignement secondaire soit effectuée par un secondaire ?

D’autres agrégés ne tiennent pas à abandonner aux primaires la direction de l'enseignement primaire lui-même. Va-t-on leur laisser la place ? Au demeurant, n'est-il pas bon que les primaires aient un chef à la culture étendue, ne seraitce que pour résister à l'influence des notables locaux ? "On ne voit pas, écrit la section de La Rochelle, ce que l'enseignement primaire gagnerait à s'isoler de l'enseignement secondaire, par l'intermédiaire duquel il reçoit un reflet des méthodes de la haute culture littéraire et scientifique "63.

L'objection la plus grave, cependant, tient à la défiance que les professeurs de lycée entretiennent vis-à-vis du contrôle de l'inspecteur d'académie. Seul un inspecteur spécialisé est censé pouvoir juger des aptitudes professorales. Les professeurs de lycée ne voient pas comment un inspecteur d'académie (ou un proviseur) agrégé de lettres peut prétendre apprécier correctement le cours d'un professeur de mathématiques. C'est d'ailleurs ce que le ministre FrançoisAlbert a objecté à la Société des agrégés : après tout, l'inspecteur d'académie

61 Association amicale des inspecteurs d'académie. Bulletin annuel, 1924, p. 8.

62 L'Agrégation, n 6, mai-juin 1925, p. 51.

63 L'Agrégation, n $^{\circ}$, mars 1926, p. 20. 
agrégé n'offre pas plus de garantie que l'inspecteur d'académie non agrégé ou primaire. La Société lui a toutefois répondu que si on nomme deux ou trois inspecteurs d'académie spécialisés auprès du recteur, on limite du moins cette non-spécialisation. Aux yeux de ses partisans, c'est d'ailleurs le principal mérite du projet d'institution d'inspecteurs régionaux : les inspecteurs d'académie des départements, non spécialisés, seraient remplacés par deux ou trois inspecteurs régionaux spécialisés (lettres, sciences, éventuellement langues vivantes) ; et ces inspecteurs, débarrassés des tâches relevant de l'enseignement primaire, auraient le temps d'inspecter tous les professeurs, qui se plaignent parfois du nombre insuffisant de visites de l'inspection générale. Le problème est que certains agrégés estiment que la spécialisation des inspecteurs régionaux serait encore insuffisante, sauf à nommer un inspecteur régional pour chaque spécialité ; mais dans ce cas mieux vaudrait augmenter le nombre des inspecteurs généraux, car les inspecteurs régionaux feraient double emploi et leurs appréciations poseraient le problème de l'inégalité de traitement des professeurs selon les académies.

Devant cette contestation, l'assemblée générale de la Société vote finalement un vœu simple, mais radical, qui reprend la logique amicaliste de ses origines, mais rompt le mouvement de rapprochement avec les inspecteurs d'académie : "La Société des agrégés demande que les inspecteurs d'académie n'aient plus que des fonctions administratives, à l'exclusion de toute fonction pédagogique, et se prononce pour un renforcement de l'inspection générale (nombre et spécialisation) "64. Ainsi les inspecteurs d'académie deviendraient-ils de simples administrateurs et n'inspecteraient-ils plus les professeurs. Le chef des agrégés devant être un agrégé, la Société n'en demande pas moins que l'inspecteur d'académie, même réduit à son rôle administratif, soit un agrégé.

Il est clair qu'un tel vœu ne saurait agréer aux inspecteurs d'académie. É. L. Ferrère fait lui-même observer que le comité des inspecteurs d'académie, présidé par le recteur, établit la liste des professeurs proposés pour les fonctions administratives et pour les promotions au choix. S’il ne connait pas les professeurs, il ne pourra faire que des propositions arbitraires (ce que les professeurs prétendent empêcher) ; et, s'il ne fait pas de propositions, le recteur tiendra compte du seul avis du proviseur, ce qui signifie que le contexte local jouera un rôle prédominant. De toute façon, remarque Ferrère, si on cantonne 
l'inspecteur d'académie à "l'inspection de l'Économat ", il ne rime à rien d'exiger des agrégés, aussi bien mettre des primaires, qui coûtent moins cher ${ }^{65}$.

On peut penser que ces hésitations sont en fait la conséquence de la doctrine de la Société des agrégés au sujet des compétences professionnelles respectives des professeurs et des membres de l'administration.

\section{2 - Grade professionnel et compétences professionnelles}

Lors de l'assemblée générale de la Société des agrégés de 1927, le communiste Georges Politzer s'exclame : "Défendons l'agrégation d'un point de vue professionnel. Sinon, nos protestations n'auront aucune portée : évitons de brimer les primaires " ${ }^{66}$. Cette injonction lui vaut des protestations et des interruptions. Elle n'en pose pas moins la question des compétences professionnelles.

En 1916, contre l'élargissement aux primaires du recrutement des inspecteurs d'académie, le président de la Fédération nationale des professeurs de lycée écrivait : "On ruine l'autorité administrative quand on ne l'établit pas sur l'autorité professionnelle "67. Il liait ainsi son opposition à des raisons de compétence. Mais c'était assimiler, implicitement, compétences administratives et compétences scientifiques.

Cette opinion est celle de la Société des agrégés. Contre les professeurs chargés de cours (licenciés), elle a été en effet amenée à élaborer une doctrine pédagogique qui fait du plus savant le meilleur professeur. Elle ne voit donc pas d'autres compétences que disciplinaires. De ce fait, elle ne reconnait d'autorité légitime qu'à l'inspection générale. Le contrôle de l'inspecteur d'académie, comme celui du proviseur, ne lui parait pas valable, dans la mesure où elle pense impossible de distinguer appréciations générales et appréciations pédagogiques. Quand, pour défendre le décret du 27 janvier 1925, le directeur de l'enseignement primaire lui représente que " ce sont surtout les qualités constantes et permanentes du personnel enseignant, c'est-à-dire l'autorité, l'habileté, le caractère, que les administrateurs locaux doivent apprécier, les qualités scientifiques étant plutôt du ressort de l'inspection générale ", la délégation de la Société des agrégés lui rétorque " qu'il est pratiquement impossible de faire le départ entre appréciations administratives et appréciations péda-

65 L'Agrégation, n 8 , juillet 1927, p. 40.

66 L'Agrégation, ${ }^{\circ}$ 6, avril-mai 1927, p. 17.

67 BOFNPL, n 98, mars-avril-mai 1916, p. 187. 
gogiques " ${ }^{68}$. De ce fait, il n'est pas question d'évoquer l'existence de qualités de jugement, de sagacité ou de discernement qui seraient utiles aux membres de l'administration.

On peut toutefois se demander si cette position n'est pas en contradiction avec le souci qu'a la Société d'accueillir les membres de l'administration et de valoriser les agrégés qui ont quitté l'Université en organisant tous les ans un " banquet des agrégés ". Car cette démarche suggère que l'agrégation mène à tout, que les agrégés sont capables de tout faire. Par conséquent, si vraiment l'agrégation est non seulement un concours de spécialiste, mais aussi et avant tout une épreuve de haute culture, la Société des agrégés, en refusant à l'inspecteur d'académie le droit de contrôle sur ses pairs, ne diminue-t-elle pas la valeur que confère, d'après elle, l'agrégation ? Faute de vouloir analyser les compétences acquises par le concours de l'agrégation, la Société des agrégés hésite donc entre, d'une part, l'affirmation de compétences professionnelles liées à la spécialisation et, d'autre part, la valorisation d'une culture générale qui rendrait apte à tout.

Son attitude la conduit à nier l'existence de qualités de jugement étrangères au jugement scientifique et à ignorer l'existence d'autres qualités, administratives. Cette position la sépare bien sûr de l'Association amicale des inspecteurs d'académie, qui considère (et a tout intérêt à considérer) que le bon administrateur possède non seulement de bonnes compétences scientifiques et pédagogiques, mais aussi quelques autres qualités. Pour son président, Aubin, en 1923, "les qualités d'esprit et de caractère nécessaires au bon administrateur sont aussi difficiles et rares que les qualités qu'on réclame d'un savant. En réalité, quoi qu'on en dise, ces qualités ne s'opposent point. Les meilleurs inspecteurs ont toujours été de bons professeurs, et on ne pourrait pas citer d'exception; et si de bons professeurs ont échoué dans les fonctions administratives, c'est que celles-ci exigent toutes les qualités qui font les bons professeurs, et d'autres qualités encore "69. Paul Lapie va encore plus loin quand il déclare à la Société des agrégés qu'à ses yeux " les qualités qu'on exige d'un administrateur départemental sont surtout des qualités de jugement et de caractère, souvent indépendantes des titres et des grades". Jugement au demeurant partagé par É. L. Ferrère lui-même, en dépit de sa position de membre du comité de la Société des agrégés :

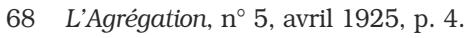

69 Association amicale des inspecteurs d'académie. Bulletin annuel, 1923, p. 17. 
" J'ai le sentiment que lorsqu'un inspecteur d'académie agrégé réussit [...] à donner à son personnel des directives utiles, à établir une liaison efficace entre l'enseignement primaire et l'enseignement secondaire, à être l'arbitre impartial entre l'autorité administrative et les syndicats, à combattre avec succès l'influence néfaste de la politique dans la marche du service dont il est chargé, il le doit à ses qualités personnelles, à son expérience, à son tact, à son énergie, beaucoup plus qu'à sa culture supérieure, beaucoup plus qu'à son titre d'agrégé " ${ }^{70}$.

Ferrère avoue qu'avant d'être nommé inspecteur d'académie, il pensait l'inverse, comme la plupart des agrégés, mais que, depuis, il a changé d'avis. Sa position demeure cependant qu'il faut distinguer inspection secondaire régionale et direction départementale de l'enseignement secondaire : si, comme la Société des agrégés, il veut que les inspecteurs d'académie soient agrégés, c'est pour en faire des inspecteurs pédagogiques régionaux. S'il s'agit d'inspection pédagogique, il faut des compétences scientifiques ; sinon, ce n’est pas la peine de recruter des agrégés.

Les débats autour du décret Lapie ne sauraient être compris hors du contexte du mouvement pour l'école unique. Aux yeux des pouvoirs publics, la circulaire du 29 septembre 1924 sur les professeurs des classes élémentaires comme le décret Lapie sont des premiers pas vers la mise en place de l'école unique. C'est au reste ce qui mécontente les professeurs de lycée, qui estiment qu'on prend les choses à l'envers ${ }^{71}$ : alors que la commission de l'école unique n'a pas encore rendu ses conclusions, des mesures sont déjà décidées, qui favorisent les primaires. On commence par les questions de personnels avant même d'avoir réglé les principes.

Nombreux sont les professeurs de l'enseignement secondaire qui s'affirment partisans de l'élargissement de la clientèle des lycées, mais qui redoutent en revanche que le mouvement pour l'école unique ne débouche sur leur éviction par les primaires et sur l'" amputation " de l'enseignement secondaire, dont la nature est pourtant, à leurs yeux, d'être un enseignement long d'imprégnation lente. Tous les professeurs, toutefois, n'en déduisent pas qu'il faut s'opposer au mouvement de rapprochement de l'enseignement primaire et de l'enseignement secondaire. Si certains refusent de dissocier inspection d'académie et direction départementale de l'inspection primaire, comme le proposent une partie des primaires et comme le préconise officiellement le Syndicat national des professeurs de lycée, c'est justement au nom de la nécessité d'une meilleure

70 L'Agrégation, n 3, décembre 1925-janvier 1926, p. 16.

$71 \mathrm{AN} \mathrm{F}^{17} 17500$. 
coordination entre les deux enseignements. Ainsi, par-delà les enjeux corporatistes, se trouvent mises à nu les divergences des professeurs de lycée au sujet de la question de l'école unique (ou du rapprochement des deux ordres d'enseignement).

$\mathrm{Au}$ demeurant, les primaires sont également divisés sur le sujet. Certains, parallèlement aux secondaires, préféreraient la voie de l'autonomie. L'épisode de l'opposition au décret Lapie présente d'ailleurs la particularité d'avoir vu le représentant d'une association de personnels de l'enseignement primaire faire des propositions à la Société des agrégés. Mais, en demandant l'autonomie du primaire par rapport aux inspecteurs d'académie et en suggérant que la coordination entre les deux enseignements soit effectuée par le seul recteur, l'Association des inspecteurs primaires anciens professeurs propose une réforme qui pourrait conduire à mettre en parallèle les écoles primaires supérieures et les lycées et faire droit à une revendication des professeurs d'écoles primaires supérieures : que leurs élèves puissent accéder à l'enseignement supérieur. Une telle perspective permettrait de contourner les lycées, mais, en maintenant deux branches parallèles du second degré, elle ne va pas dans le sens de l'école unique.

De ce point de vue, cet épisode rappelle aussi que le particularisme culturel n'est pas l'apanage des professeurs de lycée : si certains primaires (et en particulier certains inspecteurs primaires) réclament la séparation des fonctions d'inspecteur d'académie et de directeur départemental de l'enseignement primaire, c'est qu'ils supportent difficilement le contrôle d'un chef qui ne partage pas la même culture. Ce sentiment ne va cependant pas jusqu'à établir une hiérarchie. C'est un pas que franchissent au contraire certains professeurs de lycée, comme le suggère le slogan "qui peut le plus peut le moins ". Les débats autour du décret Lapie sont ainsi révélateurs de l'opposition de deux cultures et parfois de l'affirmation de la supériorité de celle du secondaire sur celle du primaire.

Cet épisode montre aussi la hantise du déclassement qui caractérise nombre de professeurs agrégés. De là le souci de la distinction, que l'on fait reposer sur les titres. Il ne faudrait cependant pas considérer que les questions de vanité n'ont concerné que les agrégés. Ces susceptibilités, en outre, n'opposent pas seulement des secondaires à des primaires : en 1923, Paul Lapie a recommandé aux inspecteurs primaires sortis du rang de ne pas inspecter les professeurs d'écoles primaires supérieures. Mais il est de fait que la crainte du nivellement 
et la valorisation des titres conquis sont particulièrement répandues chez les enseignants du secondaire. En témoigne la virulence avec laquelle les professeurs des classes élémentaires des lycées affirment également leur particularité ; ils sont d'autant plus conduits à affirmer leur identité secondaire que celle-ci ne leur est pas toujours reconnue et qu'ils veulent montrer le pas qu'ils ont franchi en réussissant un concours spécifique ${ }^{72}$. Aussi la protestation des agrégés contre le décret Lapie doit-elle être mise en parallèle avec la protestation des professeurs des classes élémentaires des lycées contre la circulaire du 29 septembre 1924, qui les mettait sous l'autorité des inspecteurs primaires. Au demeurant, alors que l'action de la Société des agrégés s'est avérée vaine (le décret Lapie n'a pas été rapporté), celle des professeurs des classes élémentaires de lycée, soutenue par le Syndicat national des professeurs de lycée ${ }^{73}$, a abouti à la circulaire du 5 décembre 1932, qui a limité le droit d'inspection des inspecteurs primaires aux seuls instituteurs (ce qui exclut les possesseurs du certificat d'aptitude à l'enseignement dans les classes élémentaires, institué en 1881 et supprimé en 1926) ${ }^{74}$. Il est vrai que la réclamation des professeurs des classes élémentaires pouvait être entendue plus facilement, dans la mesure où il suffisait d'attendre l'extinction de leur corps...

L'opposition entre secondaires et primaires paraît parfois perdurer jusqu'à nos jours. En écho à la protestation contre le décret Lapie, on peut en effet rappeler la réticence de certains agrégés d'aujourd'hui à être inspectés par des IA-IPR (inspecteurs d'académie - inspecteurs pédagogiques régionaux) provenant de l'enseignement primaire. Selon la Société des agrégés, ces derniers se montreraient spécialement vindicatifs à l'encontre des professeurs agrégés et se feraient un malin plaisir de leur imposer leurs conceptions pédagogiques. La Société des agrégés a au reste condamné la possibilité qui a été ouverte en 1990 aux inspecteurs de l'éducation nationale (IEN, ex-inspecteurs primaires) d'accéder aux fonctions d'IA-IPR, notamment sur liste d'aptitude.

La Société, en revanche, ne s'était pas opposée, en 1964, à la création du corps des IPR. Est-ce seulement parce que, dans un contexte de massification

72 Sur lidentité professionnelle des professeurs des classes élémentaires, cf. André D. Robert, "Les professeurs des classes élémentaires des lycées et leur représentation. Crépuscule et postérité d'une idéologie catégorielle (1881-1965) ", in Pierre Caspard, Jean-Noël Luc et Philippe Savoie (dir.), Lycées, lycéens, lycéennes. Deux siècles d'histoire, Paris, INRP, 2005, p. 317-329.

73 AN F $^{17} 17500$.

74 Encore la circulaire du 22 mars 1933 précise-t-elle que l'inspecteur primaire se fera accompagner par le chef d'établissement, par qui devra ensuite transiter le rapport d'inspection. 
de l'enseignement secondaire qui rendait difficile le contrôle de l'inspection générale, il était malaisé de maintenir la position de refus qui avait prévalu dans les années 1920 ? Pas seulement : le corps des IPR est spécialisé par discipline, et non par groupe de disciplines, conformément à ce que souhaitaient ceux des agrégés qui avaient combattu dans les années 1920 l'idée d'instituer des inspecteurs d'académie régionaux. De ce point de vue, les débats qui ont suivi la publication du décret Lapie, en abordant la préhistoire du corps des IPR, mettent à jour une autre caractéristique de l'identité des professeurs de lycée : l'attachement à la culture disciplinaire, à laquelle est souvent rattachée la compétence professionnelle. De cet attachement découle la conviction, très répandue entre les deux guerres chez les professeurs de lycée, selon laquelle la formation professionnelle doit être avant tout scientifique ${ }^{75}$. L'assimilation entre culture disciplinaire et compétence professionnelle a aussi pour corollaire l'idée selon laquelle il ne saurait être question de faire le départ entre contenu d'un cours et méthodes pédagogiques. Aussi seul un inspecteur spécialisé peut-il prétendre juger de l'efficacité d'une leçon. Aux yeux des professeurs de lycée, les visites des proviseurs et des inspecteurs d'académie dans les classes n'ont donc pas la légitimité de celles des inspecteurs généraux. Il n'est pas sûr que ce point de vue ait beaucoup évolué aujourd'hui, comme en ont témoigné de récents débats sur le rôle des proviseurs dans le contrôle pédagogique.

En revanche, la Société des agrégés elle-même semble avoir abandonné l'idée que les agrégés seraient les mieux qualifiés pour exercer les fonctions administratives. Entre les deux guerres, elle tendait pourtant à faire de la réussite au concours de l'agrégation un gage de culture procurant des aptitudes à ces fonctions. Elle oscillait d'ailleurs, de ce fait, entre deux points de vue : quand il s'agissait de s'opposer aux professeurs licenciés ou de refuser l'inspection par des personnels non spécialisés, elle tendait à assimiler agrégation, compétence scientifique et compétence professorale ; quand il s'agissait de prouver l'aptitude des agrégés à exercer les fonctions administratives, voire des fonctions hors de l'Université, elle tendait à assimiler agrégation, culture générale et compétences générales, comme si ces compétences générales servaient de matrice à des compétences professionnelles quelle que soit la profession exercée.

75 Cf. Jean-François Condette, Histoire de la formation des enseignants en France (XIX ${ }^{e}$ XX ${ }^{e}$ siècles), Paris, L'Harmattan, 2007. 
Tel n'était pas le point de vue des inspecteurs d'académie. Entre eux et les professeurs agrégés, il y avait alors des convergences, voire des connivences : la plupart partageaient la même culture secondaire et étaient également attachés au maintien de l'indemnité d'agrégation, donc au maintien de la distinction entre agrégés et non agrégés. Voilà qui explique la présence importante d'inspecteurs d'académie au sein de la Société des agrégés. Mais la convergence ne pouvait être totale. Non seulement, en effet, les inspecteurs d'académie tenaient au maintien de leurs prérogatives, mais encore ils estimaient avoir des qualités supplémentaires, telle la faculté de discernement, par rapport aux professeurs de lycée (alors que beaucoup de professeurs continuaient à penser in petto que les administrateurs sont souvent d'anciens professeurs fatigués). Ce qui séparait inspecteurs d'académie et professeurs agrégés, c'est bien leur analyse des compétences professionnelles.

$\mathrm{Au}$ total, toute alliance entre agrégés et inspecteurs d'académie ou entre agrégés et inspecteurs primaires était improbable. Et dans la mesure où, entre des agrégés divisés sur la stratégie à adopter, le plus petit dénominateur commun était encore la position défensive, la Société des agrégés a fini par se borner, à la fin des années 1920, à voter un vœu selon lequel "à l'avenir, nul ne pourra être nommé inspecteur d'académie s'il n'est agrégé ${ }^{76}$. Dans la pratique, les organisations corporatives de l'enseignement secondaire se sont surtout attachées à limiter les conséquences du décret Lapie : en 1929, d'après É. L. Ferrère, il n'y avait que neuf inspecteurs d'académie issus de l'enseignement primaire (ce qui correspond, en fait, à la proportion instituée en 1919, que le décret de 1925 avait abolie). En 1936, d'après J. Effroy ${ }^{77}$, ils étaient 5 sur 86. Cette posture défensive donnait cependant aux organisations corporatives de l'enseignement secondaire une allure conservatrice. Voilà pourquoi ces organisations insistaient, contre des primaires qui se présentaient en piliers du régime politique, sur le caractère démocratique et républicain de leurs arguments. Autre élément de continuité ?

Yves VERNEUIL

IUFM Champagne-Ardenne verneuil_yves@yahoo.fr

76 L'Agrégation, n 78, 15 avril 1929, p. 255.

77 Jacques Effroy, op. cit., p. 140. 